\title{
Modelling of a micro Coriolis mass flow sensor for sensitivity improvement
}

\author{
J. Groenesteijn ${ }^{1}$, L. van de Ridder ${ }^{2}$, J.C. Lötters ${ }^{1,3}$, R.J. Wiegerink ${ }^{1}$ \\ ${ }^{1}$ MESA+ Institute for Nanotechnology, University of Twente, Enschede, The Netherlands, j.groenesteijn@utwente.nl \\ ${ }^{2}$ Laboratory of Mechanical Automation, University of Twente, Enschede, The Netherlands \\ ${ }^{3}$ Bronkhorst High-Tech BV, Ruurlo, The Netherlands
}

\begin{abstract}
We have developed a multi-axis flexible body model with which we can investigate the behavior of (micro) Coriolis mass flow sensors with arbitrary channel geometry. The model has been verified by measurements on five different designs of micro Coriolis mass flow sensors. The model predicts the Eigen frequency of the first two modes within $10 \%$ when the sensor tube is filled with air, water or iso-propyl alcohol. The complex shape and high aspect ratio of the micro channels do not allow conventional FEM modelling. Instead the Matlab package SPACAR is used, which allows to model the sensor with a limited number of elements, providing fast and accurate numerical computations. This allows optimization of channel geometry and positioning of the sensing structures. The model can also be applied to other resonating structures.
\end{abstract}

Keywords-modelling; Coriolis mass flow sensor; flexible body model;

\section{INTRODUCTION}

In recent years, the use of integrated microfluidic systems has become interesting for a wide range of (bio) chemical, medical, automotive and industrial applications. This is partly due to its potential for small, accurate, reliable and costeffective gas and liquid handling systems. In these microfluidic systems, flow sensors are generally among the key components. Previously [1], we presented a micro Coriolis type flow sensor, i.e. a micro-machined flow sensor containing a vibrating tube segment in which a mass flow is subject to Coriolis forces, capable of accurately measuring the mass flow down to a few $\mu \mathrm{l} / \mathrm{h}$, independent of the temperature or fluid properties like composition, density or specific heat. High sensitivity was achieved by using a channel diameter of approximately $40 \mu \mathrm{m}$ in combination with a thin wall of approximately $1 \mu \mathrm{m}$. In order to further optimize the sensor with respect to sensitivity to mass flow an adequate simulation model is needed. With an adequate model we also hope to reduce the pressure drop and the influence of noise sources, improve the response time, and reduce the power dissipation. Furthermore, it can be used to study the suitability of alternative sensing and actuation methods. Various methods have been developed to describe the behavior of Coriolis flow sensors $[2,3,4]$. Each of these methods has been developed for specific purposes and has different advantages. However, they also have disadvantages like limitation in geometry, computation time and proprietary code. The complex structures that can be used in microfluidic micro electro-mechanical systems (MEMS) pose extra demands on the modeling method.

\section{THE MICRO CORIOLIS MASS FLOW SENSOR}

\section{A. Operation principle}

Figure 1 shows the operation principle of a Coriolis mass flow sensor. An alternating drive current $i_{d}$ will, in the presence of a constant magnetic field $B$, cause a Lorentz force that will actuate the channel in a torsional (twist) mode around the rotational axis noted by $\omega$. A mass flow $\Phi_{m}$ through the channel will induce a Coriolis force $F_{c}$ which is proportional to the mass flow and the tubes angular velocity $\omega$. The resulting Coriolis force induces an out-of-plane swing vibration mode orthogonal to the actuation mode, with an amplitude proportional to the mass flow. The capacitive comb structures, noted by $C_{i}$, are used for read-out.

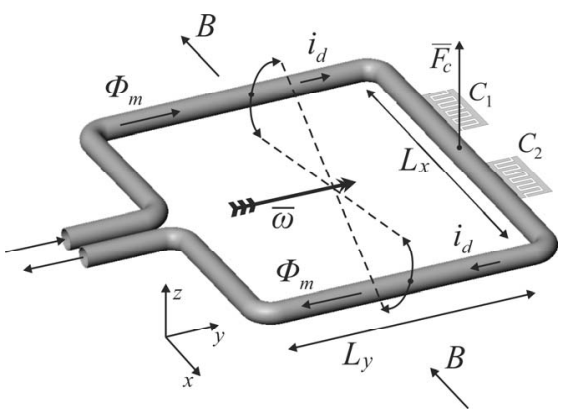

Fig. 1. Operating principle of a Coriolis mass flow sensor

Due to internal stress between the channel made of Siliconrich-nitride and the metal tracks on top of it, the channel bends slightly upwards.

\section{B. Design parameters}

The design of a Coriolis mass flow sensor is characterized by several parameters. Those parameters are the tube-shape (see Figure 1), the tube material properties, the cross-section parameters and the location of the displacement sensors.

Figure 2a shows a cross section of the channels used in the micro Coriolis mass flow sensor [1]. As can be seen, the channel is semi-circular with a flat top of the channel that is being used for metal tracks. To incorporate the properties of such a shape, a 3D representation has been made using SolidWorks[5], see Figure 2b, which gives us the second moment of inertia in the cross sectional directions. Figure $3 \mathrm{a}$ shows the ribs of the channel that are being caused by etching through the channel slits. These ribs mainly have an influence 
in the torsional stiffness of the channels. The 3D model shown in Figure $3 \mathrm{~b}$ can be used to find the relevant properties for this.

The sensors are located at equal distance from the rotational axis $\omega$, for a differential measurement. Then, the phasedifference between the two measurement signals is proportional to the mass flow.
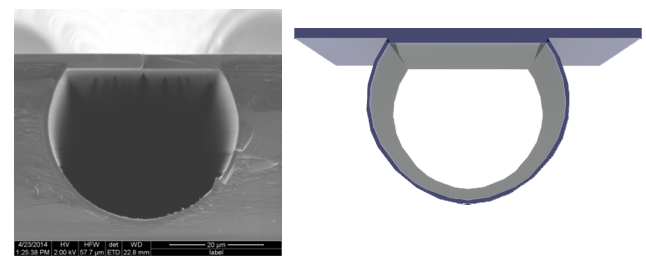

Fig. 2. Cross section of the micro-machined surface channel. Left: SEM images. Right: Modelled representation

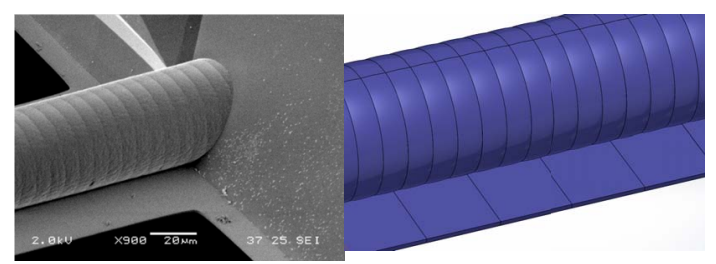

Fig. 3. The underside of the channel wall. Left: SEM image. Right: Modelled representation

\section{MODEL}

The complex shape and high aspect ratio of the used micro channels do not allow conventional Finite Element Method (FEM) modeling. Instead the Matlab package SPACAR is used [6]. This package allows modeling the sensor with a limited number of elements, providing fast and accurate numerical computations.

In the multi-body modelling approach, long, slender tubeelements are used [7]. Each element has only 6 degrees of freedom, namely the deformations, representing the strain, torsion and bending of the element. Further in the formulation, the flow induced effects, the induced forces due to the Coriolis and centrifugal acceleration of the flow, are included.

The used parameters of the model contain the material properties $\mathrm{E}$ and $\mathrm{G}$, the tube shape width, height and bending radius; the section parameters density, thickness and radius; and the fluid density. The parameters are determined using the SolidWorks model, as shown in Figure 2 and 3.

The output of the model are the mass matrix $\mathrm{M}$, the velocity sensitive matrix $\mathrm{C}$ and the stiffness matrix $\mathrm{K}$. In the model, the damping is omitted. The matrix $\mathrm{C}$ is skew-symmetric and is proportional to the mass-flow. It contains the terms induced by the flow due to the Coriolis effect.

Solving the complex eigenvalue problem

$$
\left(K+j \omega_{i} C-\omega_{i}^{2} M\right) v_{i}=0
$$

results in eigenvalues $\omega_{i}$ and the corresponding mode shapes $v_{i}$. The real part of the modeshape is the conventional mode for zero mass-flow, while the imaginary part is the Coriolis induced part. In Figure 4, the first three mode shapes for $\Phi_{m}=0$ are depicted. The first mode is the Coriolis mode, the second mode is an in-plane mode and the third is the actuation mode.

a)

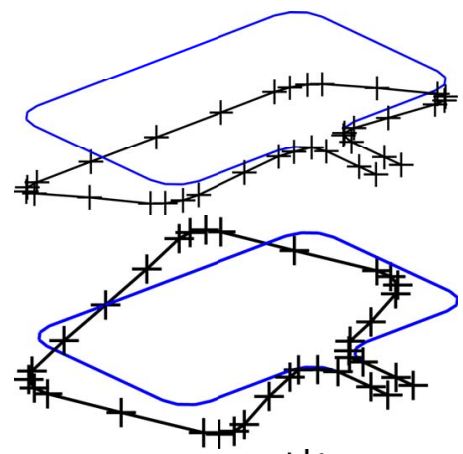

c)

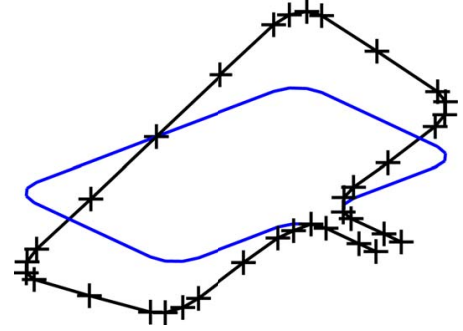

Fig. 4. Visualisation of the first three modeshapes of a Coriolis mass flow sensor with rectangular tube window with 34 tube-elements

To measure the mass-flow, the phase-difference between the sensors $s_{1}$ and $s_{2}$ is determined. Based on the model, the phase difference is equal to:

$$
\Delta \varphi=\varphi_{1}-\varphi_{2} \approx 2 \frac{\operatorname{Im}\left(s_{1}+s_{2}\right)}{\operatorname{Re}\left(s_{1}-s_{2}\right)},
$$

with $s_{i}=\Gamma_{\mathrm{s}_{\mathrm{i}}} v_{3}$, where $\Gamma_{\mathrm{s}_{\mathrm{i}}}$ is the vector of the displacement of the $i^{\text {th }}$ sensor with respect to the degrees of freedom of the model and the complex vector $v_{3}$ of the third modeshape. Note that the phase-difference will be infinite when the sensors are both on the actuation rotation axis. Given the mass-flow, the sensitivity can be calculated as:

$$
S=\frac{\Delta \varphi}{\Phi_{m}}
$$

The sensitivity is dependent on the design of the Coriolis tube, but in operation it is also a function of the fluid density and temperature. Optimization of the geometry results in a higher sensitivity and thus improvement of the accuracy of the flow sensor.

\section{MEASUREMENTS}

Figure 5 shows photographs of three different designs of micro Coriolis mass flow sensors that have been modelled and fabricated. The designs with rectangular and u-shaped tubewindow in Figure $5 \mathrm{a}$ and $5 \mathrm{~b}$ have been fabricated using two different dimensions for the width and height of the window. An overview of the dimensions of the different designs is given in Table 1. Table 2 shows the parameters of the surface channels that are common for all designs and that are used in the model. 


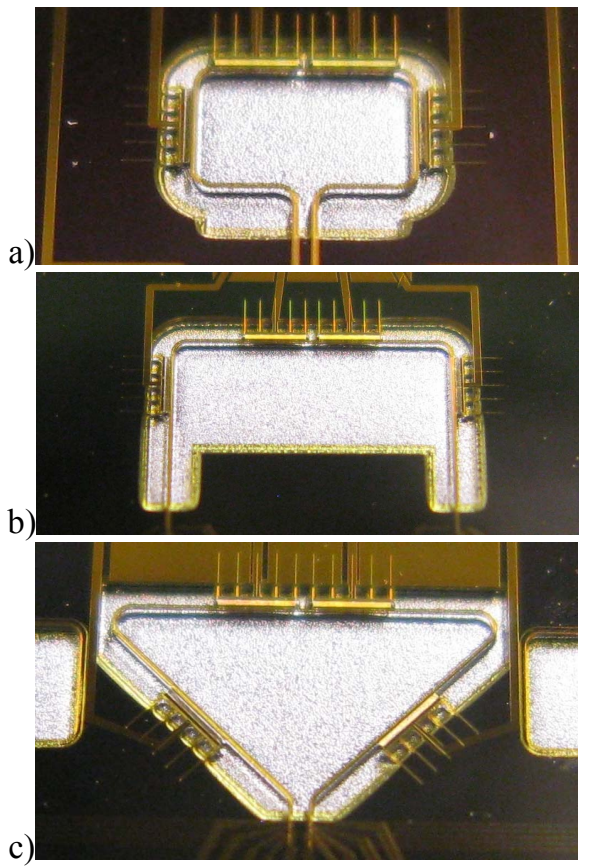

Fig. 5. Photographs of micro Coriolis mass flow sensors with a rectangular (a), a u-shaped (b) and a triangular (c) tube window.

TABLE I. DESCRIPTION OF THE FIVE DIFFERENT DESIGNS USED FOR VERIFYING THE MODEL

\begin{tabular}{|c|c|c|c|c|}
\hline$\#$ & $\begin{array}{c}\text { Tube } \\
\text { window }\end{array}$ & $\begin{array}{c}\text { Width } \\
(\mathbf{m m})\end{array}$ & $\begin{array}{c}\text { Height } \\
(\mathbf{m m})\end{array}$ & $\begin{array}{c}\text { Sensor } \\
\text { distance }(\mathbf{m m})\end{array}$ \\
\hline 1 & Rectangular & 4 & 2.5 & 1.100 \\
\hline 2 & Rectangular & 2.3 & 1.4 & 1.036 \\
\hline 3 & U-shaped & 4 & 2.5 & 1.075 \\
\hline 4 & U-shaped & 2.3 & 1.3 & 1.050 \\
\hline 5 & Triangular & 4.3 & 2.5 & 1.075 \\
\hline
\end{tabular}

TABLE II. DIMENSIONS OF THE FABRICATED CHANNELS

\begin{tabular}{|l|l|}
\hline \multicolumn{1}{|c|}{ Parameter } & \multicolumn{1}{|c|}{ Value } \\
\hline Channel width & $40 \mu \mathrm{m}$ \\
\hline Channel height & $32 \mu \mathrm{m}$ \\
\hline Thickness of the channel wall & $0.8 \mu \mathrm{m}$ \\
\hline Thickness of the flat top of the channel & $3.1 \mu \mathrm{m}$ \\
\hline Thickness of the flaps next to the channel & $2.1 \mu \mathrm{m}$ \\
\hline $\begin{array}{l}\text { Thickness of the metal actuation- and read-out } \\
\text { tracks }\end{array}$ & $250 \mathrm{~nm}$ \\
\hline Rib pitch & $8 \mu \mathrm{m}$ \\
\hline
\end{tabular}

\section{A. Eigenmodes}

Using the model, the eigenfrequency and mode shape of all eigenmodes can be examined. However, due to the limitations of the Lorentz force actuation used in the sensor, only a few of the out-of-plane modes can be actuated and measured. The two modes of most interest, the actuated twist mode and the
Coriolis force induced swing mode, are the third and first eigenmodes of the system respectively. The second mode is an in-plane swing mode that cannot be actuated and a comparison can thus not be made. Since the eigenfrequencies are heavily depending on the total mass of the free-hanging channel and the fluid inside, the measurements have been repeated using air, iso-propyl-alcohol (IPA) and water. Figure 6 shows the first (left) and third (right) eigenmodes of design 2, measured using laser Doppler vibrometry.

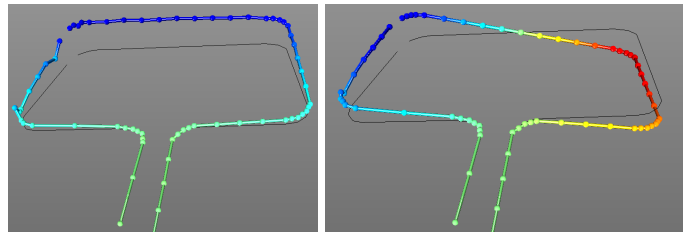

Fig. 6. Visualization of the measured swing (left) and twist (right) modeshapes of design 2. Measured using laser Doppler vibrometry.

Figure 7 and 8 show the measured and simulated eigenfrequencies of the swing mode and twist mode respectively. The simulated value is within $10 \%$ of the measured value for all designs and all fluids.

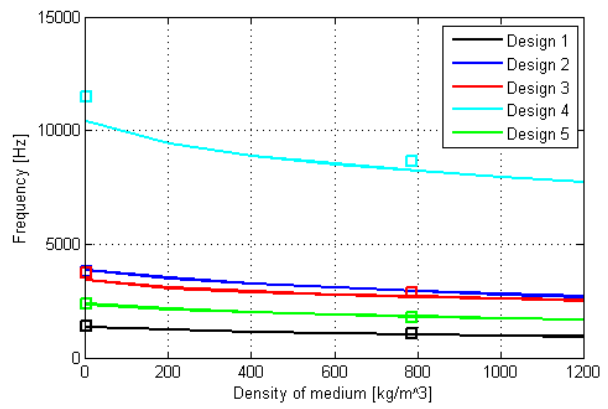

Fig. 7. Measured (squares) and modelled (lines) Eigenfrequencies of the swing mode as a function of fluid density.

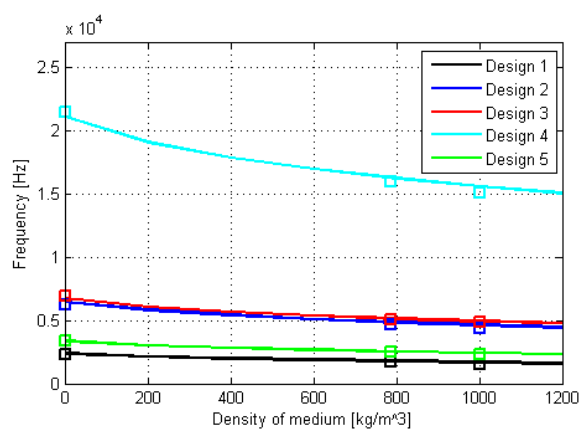

Fig. 8. Measured (squares) and modelled (lines) Eigenfrequencies of the twist mode as a function of fluid density.

\section{B. Sensitivity}

To compare the modelled sensitivity to the measurements, a mass flow of water is applied to the sensors. Lock-in amplifiers (Stanford Research SR-830) are used to measure the phase- 
shift between the signals of the two capacitive read-out structures. The results shown in Table III are measured using a mass flow between 0 and $5 \mathrm{~g} / \mathrm{h}$.

TABLE III. COMPARISON OF THE SENSITIVITY TO MASS FLOW (DEGREES/(GRAM/HOUR)) FOR WATER

\begin{tabular}{|l|l|l|l|}
\hline Design & $\begin{array}{c}\text { Measured } \\
(\% /(\mathbf{g} / \mathbf{h}))\end{array}$ & $\begin{array}{c}\text { Modelled } \\
(\% /(\mathbf{g} / \mathbf{h}))\end{array}$ & Error \\
\hline 1 & 2.97 & 2.61 & $12.1 \%$ \\
\hline 2 & 1.57 & 1.33 & $15.3 \%$ \\
\hline 3 & 0.96 & 0.85 & $11.8 \%$ \\
\hline 4 & 0.36 & 0.31 & $16 \%$ \\
\hline 5 & 3.47 & 2.58 & $25.6 \%$ \\
\hline
\end{tabular}

Here, it should be taken into account that the model does not incorporate the influence of the capacitive read-out structures. Due to stress between the Silicon-rich-nitride channel and the metal tracks on top of it, the channel bends slightly upwards. This means that the comb-fingers attached to the tube are located higher than those attached to the substrate, see Figure 9. Depending on the channel geometry, this can be several $\mu \mathrm{m}$.

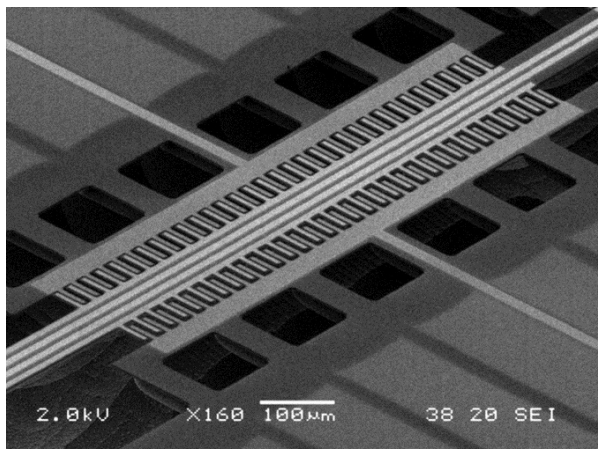

Fig. 9. SEM image of the comb-structures. Due to internal stress, the channel is slightly above the structures attached to the substrate.

Figure 10 shows the calculated capacitance between the comb-fingers as a function of the position of the channel with respect to the silicon surface. It can be seen that when the comb-fingers are on the same level and when they are far away, the $\frac{\partial C}{\partial z}$ is low, indicating a low sensitivity to those displacements. This also means that the electrical read-out is least sensitive to the largest movement, in this case, the actuated twist mode. Since the actuation and Coriolis mode are orthogonal, the Coriolis mode will reach its maximum amplitude around $\mathrm{z}_{0}$, where the electrical read-out is most sensitive to movement. The measured phase-shift is proportional to the ratio of the amplitudes of both modes. In effect, the capacitive read-out decreases the amplitude of the actuation mode, changing the ratio in favour of the Coriolis mode, increasing the measured phase-shift of the read-out. This also means that the measured sensitivity will be higher than the modelled sensitivity since this effect has not yet been incorporated in the model.

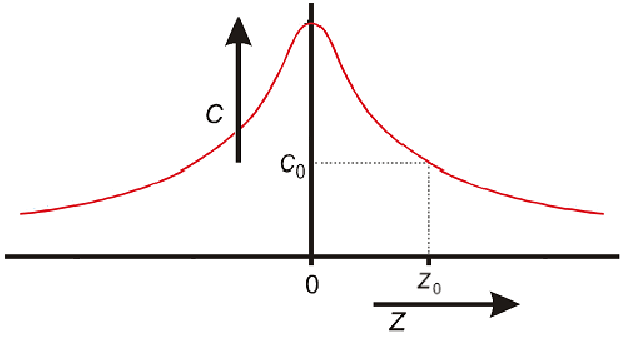

Fig. 10. Calculated capacitance between two comb-shaped electrodes as a function of the position of the channel with respect to the silicon surface in vertical direciton. The exact capacitance and $\mathrm{z}_{0}$ depend on channel and comb geometry.

\section{CONCLUSIONS}

A multi-axis flexible body model has been presented that can be used to predict the behaviour of (micro) Coriolis mass flow sensors with arbitrary channel geometry. Measurements have been performed on five different designs of micro Coriolis mass flow sensors to validate the model. Physical and mechanical parameters of the complex channel structures have been obtained from 3D representations in SolidWorks. The model has been used to find the Eigen frequency of all five designs while the channels are filled with fluids of different densities. The eigenfrequency of all measured designs and fluids are within $10 \%$ of the model. The modelled sensitivity to mass flow is lower than the measured sensitivity due to nonlinearity in the capacitive read-out structures which is not yet incorporated in the model.

The next step will be to perform an optimization algorithm on the tube shape and size and the location of the sensing elements to maximize the sensitivity to mass flow and to extend the model to include other parameters like noise and pressure drop.

\section{ACKNOWLEDGMENT}

This research is being carried out within the Coriolis Based SAS project of NanoNext NL.

\section{REFERENCES}

[1] J. Haneveld et al., Modelling, design, fabrication and characterization of a micro Coriolis mass flow sensor, J. Micromech. Microeng., 20 (2010), 125001

[2] W. B. J. Hakvoort et al., Modeling a coriolis mass flow meter for shape optimization, The 1st Joint International Conference on Multibody System Dynamics, Finland, 2010

[3] Wang S et al., The virtual Coriolis flow meter: A tool for simulation and design, Proc Instn Mech Engrs, Part C, J Mech Eng Sci 2006;220(6):817-3

[4] J. Ruoff et al., Finite element modelling of Coriolis mass flowmeters with arbitrary pipe geometry and unsteady flow conditions, Flow Measurement and Instrumentation, In Press (2014)

[5] http://www.solidworks.com

[6] http://www.wa.ctw.utwente.nl/software/spacar

[7] J.P. Meijaard, Fluid-conveying flexible pipes modeled by largedeflection finite elements in multibody systems, J. Comput. Nonlinear Dynam. 9 (2013), 01 